\title{
Retinal functional changes measured by frequency-doubling technology in patients treated with hydroxychloroquine
}

\author{
Lucia Tanga • Marco Centofanti • Francesco Oddone - Mariacristina Parravano • \\ Vincenzo Parisi • Lucia Ziccardi • Barbara Kroegler • Roberto Perricone • \\ Gianluca Manni
}

Received: 1 September 2010 /Revised: 20 December 2010 /Accepted: 29 December 2010 /Published online: 21 January 2011

(C) Springer-Verlag 2011

\begin{abstract}
Background Antimalarial drugs such as chloroquine (CQ) and hydroxychloroquine (HCQ) are mainly used in the treatment of rheumatologic diseases, and their use may be associated with irreversible retinal toxicity. Previous studies indicate early paracentral visual field loss (Humphrey 10-2) in patients taking HCQ". These paracentral defects appear before changes in other clinical parameters as visual acuity and fundoscopy. The mechanism of CQ toxicity remains unclear. It was reported that toxic doses of CQ administered for as long as 4.5 years to Rhesus monkeys caused an initial dramatic effect on ganglion cells, followed later by photoreceptors and RPE degeneration. The purpose of this study is to explore early retinal functional changes measured by
\end{abstract}

The authors have no financial relationships.

The authors have the full controls off all primary data and are agree to allow Graefe's Archives for clinical and Experimental Ophthamology to review our data.

L. Tanga $(\bowtie) \cdot M$. Centofanti $\cdot$ F. Oddone $\cdot$ M. Parravano $\cdot$

V. Parisi $\cdot$ L. Ziccardi $\cdot$ G. Manni

Fondazione G.B. Bietti for Study and Research in Ophthalmology,

IRCCS,

Via Livenza 3,

Rome 00198, Italy

e-mail: luciatanga@libero.it

M. Centofanti $\cdot$ G. Manni

UOSD Glaucoma, University of Tor Vergata,

Viale Oxford 81,

Rome 00133, Italy

B. Kroegler $\cdot$ R. Perricone

U.O.C. Reumatologia, Policlinico Tor Vergata,

Viale Oxford 81, 00133 Rome, Italy frequency-doubling technology (FDT) in patients treated with hydroxychloroquine (HCQ).

Methods Forty-eight eyes of 48 subjects treated with hydroxychloroquine (HCQ), with no signs of retinal toxicity, and 36 eyes of 36 age and sex-matched healthy subjects were enrolled in this cross-sectional, prospective, observational, case control study. Functional testing included frequency-doubling Humphrey-matrix perimetry (FDP), white-on-white Humphrey visual field perimetry (HFA), using the 24-2 and 10-2 threshold programs, multifocal electroretinogram (mfERG, Veris 4.9) and low contrast sensitivity (CS) measurement.

Results FDP mean deviation (MD) was found to be significantly reduced in HCQ-treated patients compared to controls both in the $24-2(-1.38 \pm 2.41 \mathrm{~dB}$ vs $0.21 \pm 1.83 \mathrm{~dB}$, $p<0.01)$ and in the $10-2$ program $(-0.97 \pm 2.88 \mathrm{~dB}$ vs $0.15 \pm$ $1.72 \mathrm{~dB}, p<0.01$ ). FDP pattern standard deviation (PSD) was found to be significantly worse in HCQ-treated patients compared to controls both in the $24-2(2.70 \pm 0.65 \mathrm{~dB}$ vs $2.41 \pm 0.31 \mathrm{~dB}, p<0.01$ and in the $10-2$ program $(2.86 \pm$ $0.48 \mathrm{~dB}$ vs $2.48 \pm 0.39 \mathrm{~dB}, p<0.01)$. HFA PSD and CS was also significantly reduced in HCQ patients, while response amplitude densities (RAD) were similar between patients and controls. A statistically significant difference in the ratio of the $5^{\circ}-10^{\circ} \mathrm{RAD}$ and the $0^{\circ}-2.5^{\circ} \mathrm{RAD}(0.31 \pm 0.08$ vs $0.36 \pm 0.07$ respectively, $p<0.05)$ was found between groups.

Conclusion Frequency doubling perimetry could be useful to detect early retinal impairment in patients treated with hydroxychloroquine.

Keywords Frequency doubling perimetry $\cdot$ Retinal toxicity $\cdot$ Hydroxychloroquine 


\section{Introduction}

Chloroquine (CQ) and hydroxychloroquine (HCQ) are currently used in the treatment of malaria and in treatment of rheumatologic diseases such as rheumatoid arthritis, systemic lupus erythematosus, sarcoidosis, dermatomyositis and $\mathrm{Sjögren} \mathrm{disease} \mathrm{[1].}$

Retinal toxicity represents the major and potentially most serious irreversible side-effect of this type of treatment, occurring in approximately 10-20\% of patients who received CQ and in 3\% of patients who received HCQ therapy [2-5].

Hystological analysis of human and animal retinas with CQ toxicity has shown multilamellar structures through the retina, followed by loss of retinal ganglion cells (RGCs), photoreceptors and retinal pigment epilthelium (RPE) atrophy. The accumulation of these multilamellar structures was thought to result either from inhibition of lysosomal phospholipases or from inhibition of protein synthesis $[6,7]$

Funduscopically, the early stages of retinal toxicity can be characterized as a reversible loss of foveal reflex, followed by a development of abnormalities of the retinal pigmented epithelium (RPE) associated with paracentral scotomas, and, in the advanced stage, by the classical bull's eye maculopathy, associated with a central scotoma and visual acuity (VA) loss [8, 9].

Anatomically, a retinal nerve fiber layer (RNFL) loss has been shown in patients under long-term CQ treatment compared to healthy subjects $[10,11]$.

Nevertheless, the identification of early functional retinal impairment of $\mathrm{CQ} / \mathrm{HCQ}$ retinal toxicity appears to be difficult before the development of anatomic changes at fundoscopy and symptomatic loss of vision.

Examination tests that are commonly used in evaluation of $\mathrm{CQ} / \mathrm{HCQ}$ retinal toxicity include Amsler grid, VA testing, ophthalmoscopy and visual field testing $[9,12$, 13].

Standard achromatic perimetry is considered more sensitive in the early detection of functional changes than VA, color test and retinal changes at fundoscopy [13, 14].

Functional retinal changes, such as contrast sensitivity [14] and multifocal electroretinographic (mfERG) response abnormalities [15, 16], have been detected at early stages in HCQ-treated patients, even in the preclinical phases.

Recently, frequency-doubling perimetry (FDP) has been introduced as a visual field testing technology that allows selective stimulation of the low redundant magnocellular sub-population of ganglion cells, allowing early detection of glaucoma functional loss [17].

The aim of this study is to explore early retinal functional changes measured by frequency-doubling technology (FDT) in patients treated with HCQ.

\section{Material and methods}

Patients treated with HCQ for rheumatological diseases were included in this cross-sectional case-control study. Healthy subjects were included in the control group. The study was carried out in accordance with the Declaration of Helsinki, was approved by the ethics committee of the institution and written informed consent was obtained from all enrolled subjects.

The inclusion criteria for patients included: treatment with HCQ for at least 3 months, age $>18$ years and transparent ocular media.

Control subjects needed to meet the following inclusion criteria: no history of treatment with HCQ, age $>18$ years and clear lens.

The exclusion criteria were the same for both groups: spherical refractive error $> \pm 6$ diopters, astigmatism $> \pm 3$ diopters, any active or past retinal pathologies (including diabetic retinopathy or age-related macular degeneration), diagnosis of glaucoma or ocular hypertension (intraocular pressure $>22 \mathrm{mmHg}$ ), opacities of optic media that could bias functional and structural retinal testing, history of ocular surgery.

All subjects underwent a complete ophthalmologic examination including: best-corrected visual acuity (BCVA) with Early Treatment Diabetic Retinopathy Study (ETDRS) charts at 4 meters, contrast sensitivity (CS) with MultiContrast Sloan Letter Flip Book at 2 meters testing over a range of contrasts to $100 \%, 25 \%, 10 \%, 5 \%, 2,5 \%, 1,25 \%$. Goldmann applanation tonometry, slit-lamp examination and indirect ophthalmoscopy were also performed. Functional testing included frequency-doubling Humphrey-matrix perimetry (FDP, Carl Zeiss Meditec), white-on-white Humphrey visual field perimetry (HFA, Carl Zeiss Meditec, Dublin, CA, USA), and multifocal electroretinogram (mfERG, Veris 4.9). The primary objective was to explore by FDP a possible retinal sensitivity reduction in HCQ-treated patients. Secondary objectives included the evaluation of additional functional retinal changes at HFA and $\mathrm{mfERG}$, and the influence of the duration of the therapy on the functional retinal impairment.

Functional testing

All enrolled subjects performed two FDP tests within 2 weeks using the 24-2 and 10-2 threshold program, in order to assess the test-retest variability and to rule out a relevant learning curve [18]. In addition, subjects performed two white-on-white 24-2 and 10-2 SITA standard HFA tests. Reliability criteria were defined as fixation errors $<15 \%$, false-positives and false-negatives $<15 \%$. If the second test was unreliable, a third exam was performed and considered for statistical analysis. In the case of a third 
unreliable test, the subject was excluded from the study. Mean deviation (MD) and pattern standard deviation (PSD) values were considered for the analysis.

Contrast sensitivity was performed with Multi-Contrast Sloan Letter Flip Book at 2 meters.

These charts are retroilluminated, presented in a dark room, and allow testing over a range of contrasts $(1.25 \%$, $2.5 \%, 5 \%, 10 \%, 25 \%$, and $100 \%)$. They use a logarithmic progression of letter size ( 0.1 per row), a constant number of letters per row, and letters of equal legibility, making task difficulty constant regardless of the level of acuity tested. Testing was conducted monocularly in the study eye at $2 \mathrm{~m}$ under the same mesopic luminance level for all subjects. Patients were tested with the best optical correction, and results are reported as the logarithm of the logMAR.

VERIS Clinic ${ }^{\text {TM }} 4.9$ (Electro-Diagnostic Imaging, San Mateo, CA, USA) was used for mfERG assessment, using a previously published method $[19,20]$. In all patient and control eyes, mfERG was recorded in the presence of dilated pupils with $1 \%$ tropicamide.

The average response amplitude densities (RAD in nanovolt/degree [2]) between the first negative peak, N1, and the first positive peak, P1 obtained from five concentric anular retinal regions (rings) centered on the fovea were analysed. The N1-P1 RADs derived from 0 to 2.5 degrees (ring 1, R1), from 2.5 to 5 degrees (ring 2, R2), from 5 to 10 degrees (ring 3, R3), from 10 to 15 degrees (ring 4, R4) and from 15 to 20 degrees (ring 5, R5) were also investigated. Furthermore, the ratio between R3/R1 and $\mathrm{R} 2 / \mathrm{R} 1$ was calculated and compared between groups.

A signal-to-noise ratio of $\geq 3$ was accepted for mfERG measurements.

\section{Statistical analysis}

Demographic and descriptive data were expressed as mean \pm standard deviation (SD).

Normal distribution of data was assessed by the Shapiro-Wilk test. The right eye was arbitrarily chosen for statistical analysis. Frequencies of categorical variables were compared between groups by Chi-squared and Fisher's exact test as appropriate. Comparisons of continuous variables between groups were performed by independent samples $t$-test or Mann-Whitney U test as appropriate.

The relationship between functional parameters and duration of HCQ treatment was explored by linear regression analysis.

It has been reported in the literature that HCQ retinal toxicity is correlated with the dose of the drug per body weight, and the American Academy of Opthalmology considers $6.5 \mathrm{mg} / \mathrm{kg} /$ day to be the highest safe dose of treatment, at least in the first 5 years [5]. We then compared retinal sensitivity between patients that received a dose of $\mathrm{HCQ}>6.5 \mathrm{mg} / \mathrm{kg} / /$ day with those that received a dose $<6.5 \mathrm{mg} / \mathrm{kg} /$ day in our sample population.

With 84 patients enrolled in the study, the power to detect a $1.5 \mathrm{~dB}$ difference of FDP mean deviation between groups was $80 \%$, with a standard deviation of 2.4 and a type II error set at 0.05 . The analysis was performed using JMP 7.0 (SAS Institute Inc. Cary, NC, USA)

\section{Results}

Forty-eight patients treated with HCQ $(51.40 \pm 11.57$ years, range 27-69, M/F 12/36) and 36 age- and sex- matched healthy control subjects $(47.7 \pm 10.50$ years, range $27-69$, $\mathrm{M} / \mathrm{F} 7 / 29$ ) were included in the analysis.

Most of the included patients suffered from rheumatoid arthritis (91.7\%), and only four patients (8.3\%) from systemic lupus erythematosus.

Patients were treated with HCQ at a dosage of $400 \mathrm{mg} /$ day for an median time of 36 months (range 3.5 months to 156 months).

Age, BCVA and IOP values were similar between groups. (Table 1)

FDP MD was found to be significantly reduced in HCQtreated patients compared to controls, both in the 24-2 $(-1.38 \pm 2.41 \mathrm{~dB}$ vs $0.21 \pm 1.83 \mathrm{~dB}, p<0.01)$ and in the $10-2$ program $(-0.97 \pm 2.88 \mathrm{~dB}$ vs $0.15 \pm 1.72 \mathrm{~dB}, p<0.02)$. The relationship between 24-2 HFA MD and FDP MD is presented in Fig. 1.

FDP pattern standard deviation (PSD) was found to be significantly worse in HCQ-treated patients than in controls both in the $24-2(2.70 \pm 0.65 \mathrm{~dB}$ vs $2.41 \pm 0.31 \mathrm{~dB}, p<0.01)$ and in the $10-2$ program $(2.86 \pm 0.48 \mathrm{~dB}$ vs $2.48 \pm 0.39 \mathrm{~dB}$, $p<0.01$ ).

HFA PSD was found to be significantly worse in HCQ-treated patients compared to controls in 24-2 program $(1.54 \pm 0.34 \mathrm{~dB}$ vs $1.40 \pm 0.33 \mathrm{~dB}, p<0.05)$ Fig 2 .

HFA MD 24-2, HFA MD 10-2 and HFA PSD 10-2 were similar between patients and controls.

The CS was found to be significantly reduced in HCQtreated patients compared to controls at the range of

Table 1 Sample characteristics

\begin{tabular}{lcll}
\hline & HCQ group & Controls & $P$ \\
\hline$N$ & & & \\
\hline Gender (M/F) & $12 / 36$ & 36 & - \\
Continuous variables (mean $\pm \mathrm{SD})$ & $7 / 29$ & - \\
Age (years) & $51.40 \pm 11.57$ & $47.70 \pm 10.50$ & 0.12 \\
BCVA (LogMAR) & $-0.03 \pm 0.05$ & $-0.02 \pm 0.06$ & 0.41 \\
IOP (mmHg) & $14.16 \pm 2.61$ & $14.75 \pm 2.35$ & 0.48 \\
\hline
\end{tabular}




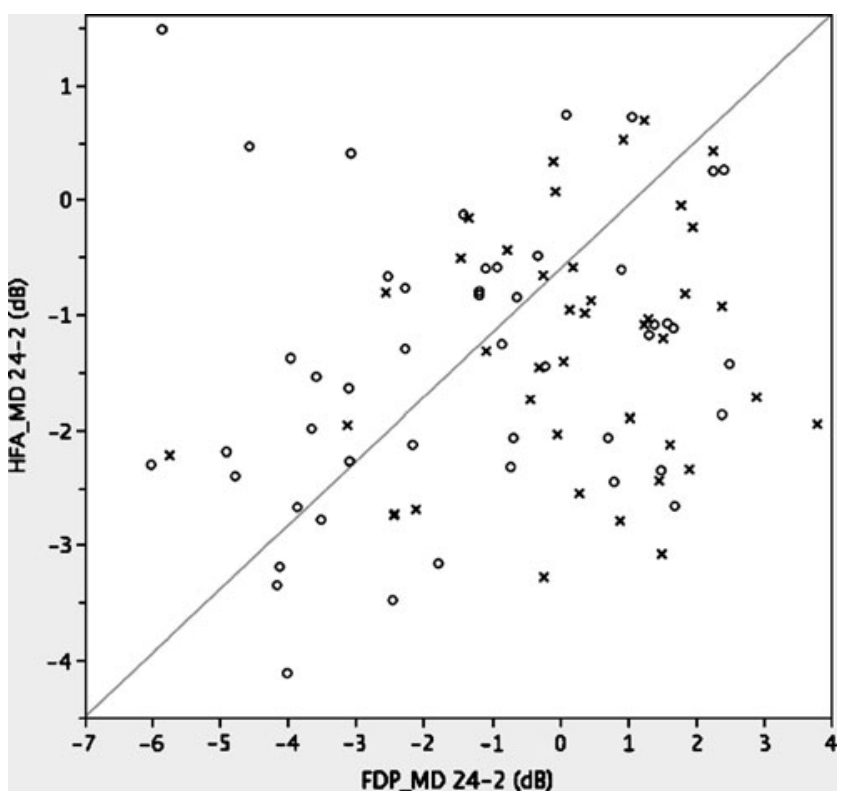

Fig. 1 Scatterplot between frequency-doubling perimetry (FDP) mean deviation (MD) and Humphrey field analyzer (HFA) MD. HCQtreated patients are indicated by circles and control subjects by crosses

contrast of $10 \%(0.09 \pm 0.07$ vs $0.04 \pm 0.10 ; p<0.02), 5 \%$ $(0.17 \pm 0.10$ vs $0.12 \pm 0.98 ; p<0.05), 2.5 \%(0.31 \pm 0.12$ vs $0.25 \pm 0.09 ; p<0.05)$.

In the mfERG no statistically significant difference was found at any ring between the two groups in the response amplitude density (RAD): R1 RAD N1-P1 (HCQ patients $84.77 \pm 26.60$ vs controls $79.31 \pm 23.81, p=0.50$ ), R2 RAD N1-P1 (HCQ patients $42.45 \pm 11.10$ vs controls $42.14 \pm 12.98$, $p=0.93$ ), R3 RAD N1-P1 (HCQ patients $25.79 \pm 7.15$ vs controls 26.73 $\pm 8.07, p=0.58$ ), R4 RAD N1-P1 (HCQ patients $17.78 \pm 5.90$ vs controls $18.68 \pm 5.83, p=0.50)$, R5 RAD N1-P1 (HCQ patients $13.85 \pm 4.02$ vs controls $13.93 \pm$ $4.23, p=0.70$ ). Furthermore, the mean amplitude ratio of $\mathrm{R} 3 /$ $\mathrm{R} 1$ and R2/R1 between the groups has been explored, and a statistically significant difference between HCQ-treated patients and controls was found for the $\mathrm{R} / 3 / \mathrm{R} 1$ ratio $(0.31 \pm$ 0.08 vs $0.36 \pm 0.07$ respectively, $p<0.05$ ), but not for the $\mathrm{R} 2 /$ $\mathrm{R} 1$ ratio $(0.51 \pm 0.09$ vs $0.54 \pm 0.09$ respectively, $\mathrm{p}=0.22)$.

No significant linear relationship was found between any functional parameters and the duration of therapy. The $\mathrm{R}$ [2] of FDP 24-2 MD and PSD versus treatment duration was $0.05(p=0.12)$ and $0.06(p=0.09)$ respectively.

Differences between functional parameters obtained with FDP, HFA and mfERG were evaluated by dividing the patients according to the duration of the HCQ therapy. Patients were divided into three groups: patients taking HCQ for less than 36 months (26 patients, group 1), patients taking HCQ for more than 36 months (22 patients, group 2) and healthy control subjects (36 patients, group 3 ). The value of 36 months was chosen as the cut-off time because it was the median value of treatment duration in our sample.
FDP and HFA parameters were found to be significantly different between each HCQ group and controls. Moreover, FDP and HFA parameters were found to be worse in patients treated for a longer time than in patients treated for ashorter time ( $<36$ months).(Tables 2,3$)$

Furthermore, FDP MD was found to be statistically significantly different between patients treated with a dosage of HCQ $>6.5 \mathrm{mg} / \mathrm{kg} / \mathrm{day}$ compared to patients treated with lower dosage $(-2.43 \pm 2.13$ vs $-0.75 \pm 1.95 \mathrm{~dB}$ respectively, $p<0.05)$. No significant differences were detected in FDP PSD ( $2.9 \pm 0.65$ vs $2.71 \pm 0.71 \mathrm{~dB}$ respectively $p=0.13)$ or HFA global indices (MD: $-1.61 \pm 1.2$ vs $1.52 \pm 1.16 \mathrm{~dB}, p=0.73$; PSD: $1.77 \pm 0.49$ vs $1.47 \pm 0.25 \mathrm{~dB}$ respectively, $p=0.08$ ).

\section{Discussion}

This study supports previous knowledge of an early retinal functional impairment in HCQ-treated patients without any clinical retinal abnormalities.

We found that HCQ-treated patients, without any clinically detectable RPE abnormalities, showed a decreased threshold of retinal sensitivity as measured by FDP and HFA. FDP mean deviation and pattern standard deviation have been found to be significantly worse in the treated patients than in control subjects. Furthermore, we found a reduction of contrast sensitivity, of HFA pattern standard deviation and of mfERG R3/R1 ring ratio.

Retinal toxicity is probably due to the inhibition of lysosomal phospholipases and/or of protein synthesis with multilamellar structures accumulation through the retina. Histologically, this accumulation is followed by retinal ganglion cell loss, photoreceptor loss and RPE atrophy [6].

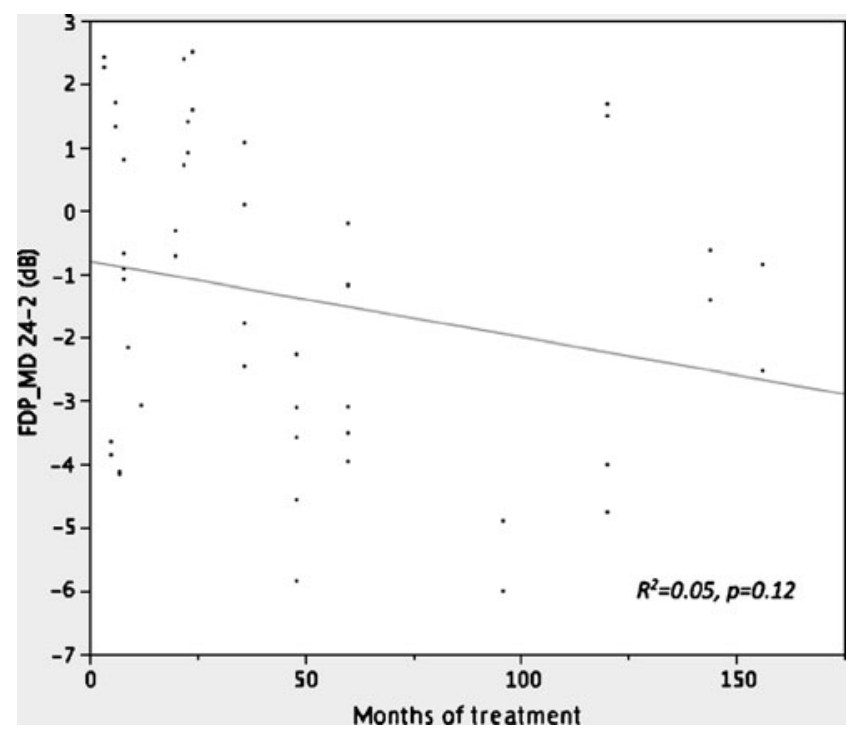

Fig. 2 Relationship between frequency-doubling perimetry (FDP) mean deviation (MD) and treatment duration (months) 
Table 2 Comparison between controls and HCQ-treated patients based on duration of therapy in Humphrey-matrix; group 1: patients taking HCQ for less than 36 months (26 patients); group 2: patients taking HCQ for more than 36 months (22 patients); group 3: healthy control subjects (36 pts)

\begin{tabular}{|c|c|c|c|c|c|c|}
\hline Humphrey-matrix & $\begin{array}{l}\text { Group } 1 \\
(\text { mean } \pm \mathrm{SD})\end{array}$ & $\begin{array}{l}\text { Group } 2 \\
(\text { mean } \pm \text { SD) }\end{array}$ & $\begin{array}{l}\text { Group } 3 \\
(\text { mean } \pm \mathrm{SD})\end{array}$ & $\begin{array}{l}\text { Group 1vs } \\
\text { group } 3(p)\end{array}$ & $\begin{array}{l}\text { Group } 2 \text { vs } \\
\text { group } 3(p)\end{array}$ & $\begin{array}{l}\text { Group } 1 \mathrm{vs} \\
\text { group } 2(p)\end{array}$ \\
\hline MD 24-2 & $-0.37 \pm 2.19$ & $-2.57 \pm 2.13$ & $0.21 \pm 1.83$ & 0.41 & $<0.01 *$ & $<0.01^{*}$ \\
\hline PSD 24-2 & $2.84 \pm 0.81$ & $2.71 \pm 0.38$ & $2.41 \pm 0.31$ & $<0.01$ & 0.06 & 0.45 \\
\hline MD $10-2$ & $0.06 \pm 2.48$ & $-2.70 \pm 2.72$ & $0.15 \pm 1.72$ & 0.98 & $<0.01^{*}$ & $<0.01^{*}$ \\
\hline PSD $10-2$ & $2.77 \pm 0.54$ & $3.01 \pm 0.33$ & $2.48 \pm 0.39$ & $0.04 *$ & $<0.01 *$ & 0.09 \\
\hline
\end{tabular}

Rosenthal et al. reported that toxic doses of CQ administered to Rhesus monkeys caused an initial dramatic effect on RGCs, followed by photoreceptor loss and RPE degeneration [7]. Hallberg et al. found that morphological and biochemical signs of phospholipidosis were evident only in the RGCs of CQ-treated mice [6]

Bonanomi et al. showed that patients under long-term CQ treatment could present a significantly reduced RNFL thickness compared to healthy subjects by means of scanning laser polarimetry GDx Nerve Fibre Analyser (software v.2.0.01), and that the RNFL loss was correlated to CQ daily dosage [11].

Clinically advanced retinal toxicity could be easily evaluated with different tests as VA, standard visual field testing and ophthalmoscopy, but those tests do not seem to detect early stages [5]. For this reason, this study explored the possible detection of early functional retinal impairment using frequency-doubling technology.

The finding of a generalized reduction of retinal sensitivity to frequency-doubled stimuli in HCQ-treated patients might be related to a higher susceptibility of the magnocellular RGCs to HCQ; this might be responsible for the early selective loss of this cell component. Nonetheless, another possible explanation is that the MGCs have similar susceptibility to HCQ compared to other RGCs, but are simply less redundant than the whole RGC population, with no overlap between receptive fields, allowing the earlier detection of MGC loss if selectively stimulated with frequency-doubled stimuli.

In this study, patients were tested both 24-2 and 10-2 programs with both HFA and FDP. The spatial and temporal characteristics of Humphrey-matrix 10-2 test do not allow to the stimulus to appear to be frequencydoubled, so the threshold determination is a flicker sensitivity response, which corresponds to a contrast sensitivity test. The changes of Humphrey-matrix 10-2 found in this study are consistent with the results of Bishara et al., who showed that contrast sensitivity is reduced in the early phases of retinal toxicity [14]. Also the low-contrast sensitivity, evaluated with Multi-Contrast Sloan Letter Flip Book at 2 meters, was found to be reduced early in HCQtreated patients in the present study.

The Humphrey-matrix parameters showed abnormalities even in the presence of normal mfERG; indeed, no statistically significant differences have been found between HCQ-treated patients and controls in the RAD in the mfERG in all rings. In the mfERG, the bioelectrical signal is derived from cones and bipolar cells, with smaller contribution from other retinal neurons. Since RGCs could be affected first, even in the absence of a defect in photoreceptor or bipolar cell function, we evaluated the abnormalities in Humphrey-matrix results that selectively study the MGCs. Early HCQ-induced changes could be detected early by detecting changes in the M-y ganglion cell population, which represents a low-redundancy system, and therefore could be followed with frequency-doubling perimetry.

It has been described in the literature that the first changes in mfERG occur in the paracentral regions, where the ratio of the rings may be affected [21]. Although the mean ring amplitudes appeared to be similar in this study between HCQ and controls, a statistically significant

Table 3 Comparison between controls and HCQ-treated patients based on duration of therapy in HFA. Group 1: patients taking HCQ for less than 36 months (26 patients); group 2: patients taking HCQ for more than 36 months (22 patients); group 3: healthy control subjects (36 pts)

\begin{tabular}{lllllll}
\hline HFA (dB) & $\begin{array}{l}\text { Group 1 } \\
(\text { mean } \pm \text { SD) }\end{array}$ & $\begin{array}{l}\text { Group 2 } \\
(\text { mean } \pm \text { SD) }\end{array}$ & $\begin{array}{l}\text { Group 3 } \\
(\text { mean } \pm \text { SD) }\end{array}$ & $\begin{array}{l}\text { Group 1vs } \\
\text { group 3 }(p)\end{array}$ & $\begin{array}{l}\text { Group 2 vs } \\
\text { group 3 }(p)\end{array}$ & $\begin{array}{l}\text { Group 1 vs } \\
\text { group 2 }(p)\end{array}$ \\
\hline MD 24-2 & $-1.46 \pm 1.30$ & $-1.37 \pm 1.17$ & $-1.31 \pm 1.06$ & 0.83 & 0.97 & 0.79 \\
PSD 24-2 & $1.48 \pm 0.27$ & $1.61 \pm 0.39$ & $1.40 \pm 0.30$ & 0.53 & $0.03 *$ & 0.18 \\
MD 10-2 & $-1.58 \pm 1.23$ & $-2.00 \pm 1.39$ & $-1.27 \pm 0.89$ & 0.54 & 0.76 & $0.02 *$ \\
PSD 10-2 & $1.09 \pm 0.22$ & $1.26 \pm 0.42$ & $1.04 \pm 0.16$ & 0.76 & 0.18 \\
\hline
\end{tabular}


difference was found in the ratio between the outer ring R3 and the inner ring R1, supporting the finding of subtle early changes of retinal function.

It should be also highlighted that more sophisticated, non-conventional methods of stimulation with mfERG have been described as detecting pre-clinical retinal function changes in HCQ-treated patients, emphasizing second-order adaptational effects such as modulated multifocal flashes with interleaved global flashes, as previously described in the paper by Penrose PJ et al. [22]

No statistically significant relationship between functional data versus treatment duration was found, as explored by linear regression analysis in the present study. Nevertheless, a difference in functional parameters between longterm treated and short-term treated patients was found, indicating that there might be a threshold effect of treatment duration on visual function change, rather than a linear continuous effect causing a continuous decay over time. Nevertheless, despite the fact that the spectrum of treatment duration was broad in our sample population (3.5 to 156 months), most of the patients were treated between 3.5 and 50 months (32/48) and only $16 / 48$ for a longer time, indicating that the lack of relationship found between functional data and HCQ treatment duration should be interpreted with caution.

Furthermore, the patients' stratification according to the duration of the therapy indicated that patients that assumed therapy for a short period of time showed only minimal retinal functional changes, compared to larger changes observed in patients taking therapy over a longer period of time. Moreover, previously published studies reported that retinal toxicity related to HCQ treatement is related to the daily dosage per $\mathrm{kg}$ of body weight, and the American Academy of Opthalmology considers $6.5 \mathrm{mg} / \mathrm{kg} /$ day to be the highest safe dose of treatment, at least in the first 5 years [5]. In our study, the prescribed dose is constant (400 $\mathrm{mg} /$ day), and we assume that patients are fully compliant, but this could be a source of error in the evaluation of the relationship between retinal toxicity and treatment duration.

The results of our study indicate that retinal sensitivity as expressed by FDP MD in patients treated with a daily dosage $>6.5 \mathrm{mg} / \mathrm{kg}$ is reduced compared to patients treated with lower daily dosages, while no differences were detected for other retinal function measures.

The Humphrey-matrix changes are present in the early phases, and they could be considered as good candidates for an early detection of the HCQ retinopathy and for its follow-up.

The differences in FDP values found in this study are moderate. This is a limitation for their use in clinical practice, even if these results are consistent with pathogenetic mechanism of HCQ retinal toxicity.
The presence of functional retinal changes in late-stage HCQ retinopathy could be related to the long duration of the HCQ therapy, but also to the long duration of the disease. In fact, a limitation of this study is that control subjects are healthy, rather than patients with rheumatic diseases but not taking HCQ.

In conclusion, functional retinal testing by frequencydoubling perimetry could be useful for the identification of early retinal impairment in HCQ-treated patients without any sign of clinically detectable retinal abnormalities, although broader diagnostic studies are required to accurately assess its sensitivity and specificity in clinical settings, for screening purposes and follow-up.

\section{References}

1. Rynes RI (1997) Antimalarial drugs in the treatment of rheumatological diseases. Br J Rheumatol 36:799-805

2. Mavrikakis I, Sfikakis PP, Mavrikakis E, Rougas K, Nikolaou A, Kostopoulos C, Mavrikakis (2003) The incidence of irreversible retinal toxicity in patient treated with hydroxychloroquine: a reappraisal. Ophthalmology 110:1321-1326

3. Levy GD, Munz SJ, Paschal J, Cohen HB, Pince KJ, Peterson T (1997) Incidence of hydroxychloroquine retinopathy in 1,207 patients in a large multicenter outpatient practice. Arthritis Rheum 40:1482-1486

4. Tzekov R (2005) Ocular toxicity due to chloroquine and hydroxychloroquine: electrophysiological and visual function correlates. Doc Ophthalmol 110:111-120

5. Marmor MF, Carr RE, Easterbrook M, Farjo AA, Mieler WF, Academy A, American Academy of Ophthalmology (2002) Recommendations on screening for chloroquine and hydroxychloroquine retinopathy: a report by the American Academy of Ophthalmology. Ophthalmology 109:1377-1382

6. Hallberg A, Naeser P, Andersson A (1990) Effects of long-term chloroquine exposure on the phospholipids metabolism in retina and pigment epithelium of the mouse. Acta Ophthalmol (Copenh) 68:125-130

7. Rosenthal AR, Kolb H, Bergsma D, Huxsoll D, Hopkins JL (1978) Choroquine retinopathy in the rhesus monkey. Invest Ophthalmol Vis Sci 17:1158-1175

8. Easterbrook M (1999) Detection and prevention of maculopathy associated with antimalarial agents. Int Ophthalmol Clin 39:49-57

9. Carr RE, Gouras P, Gunkel RD (1966) Chloroquine retinopathy. Early detection by retinal threshold test. Arch Ophthalmol 75:171-178

10. Pasadhika S, Fishman GA (2010) Effects of chronic exposure to hydroxychloroquine or chloroquine on inner retinal structures. Eye (Lond) 24:340-6

11. Bonanomi MT, Dantas NC, Medeiros FA (2006) Retinal nerve fibre layer thickness measurements in patient using chloroquine. Clin Exp Ophthalmol 34:130-136

12. Browning DJ (2002) Hydroxychloroquine retinopathy: screening for drug toxicity. Am J Ophthalmol 133:649-656

13. Elder M, Rahman AM, McLay J (2006) Early Paracentral Visual Field Loss in Patients Taking Hydroxychloroquine. Arch Ophthalmol 124:1729-1733

14. Bishara SA, Matamoros N (1989) Evaluation of several tests in screening for chloroquine maculopathy. Eye 3:777-782 
15. Maturi RK, Yu M, Weleber RG (2004) Multifocal electroretinographic evaluation of long-term hydroxychloroquine users. Arch Ophthalmol 122:973-981

16. Lai TY, Chan WM, Li H et al (2005) Multifocal electroretinographic changes in patients receiving hydroxychloroquine therapy. Am J Ophthalmol 140:794-807

17. Johnson CA, Cioffi GA, Van Buskirk EM (1999) Frequencydoubling technology perimetry using 24-2 stimulus presentation pattern. Optom Vis Sci 76(8):571-581

18. Centofanti M, Fogagnolo P, Oddone F, Orzalesi N, Vetrugno M, Manni G, Rossetti L (2008) Learning effect of humphrey matrix frequency doubling technology perimetry in patients with ocular hypertension. J Glaucoma 17:436-441

19. Holz FG, Wolfensberger TJ, Piguet B, Gross-Jendroska M, Wells JA, Minassian DC, Chisholm IH, Bird AC (1994) Bilateral macular drusen in age-related macular degeneration. Prognosis and risk factors. Ophthalmology 1101:1522-1528

20. Parisi V, Perillo L, Tedeschi M, Scassa C, Gallinaro G, Capaldo N, Varano M (2007) Macular function in eyes with early age-related macular degeneration with or without contralateral late age-related macular degeneration. Retina 27:879890

21. Lyons JS, Severns ML (2007) Detection of early hydroxychloroquine retinal toxicity enhanced by ring ratio analysis of multifocal electroretinography. Am J Ophthalmol 143(5):801880

22. Penrose PJ, Tzekov RT, Sutter EE, Fu AD, Allen AW Jr, Fung WE, Oxford KW (2003) Multifocal electroretinography evaluation for early detection of retinal dysfunction in patients taking hydroxychloroquine. Retina 23(4):503-512 
Reproduced with permission of the copyright owner. Further reproduction prohibited without permission. 